\title{
Steady State Solution and Stability of an Age-Structured MSIQR Epidemic Model
}

\author{
Meihong Qiao, ${ }^{1,2}$, Huan Qi ${ }^{1}$, Tianhai Tian ${ }^{3,4}$ \\ ${ }^{1}$ Department of Control Science and Engineering, Huazhong University of Science and Technology, Wuhan, China \\ ${ }^{2}$ School of Mathematics and Physics, China University of Geosciences, Wuhan, China \\ ${ }^{3}$ School of Mathematical Sciences, Monash University, Melbourne, Australia \\ ${ }^{4}$ Department of Mathematics, University of Glasgow, Glasgow, UK \\ E-mail:qihuan@mail.hust.edu.cn
}

Received March 19, 2010; revised April 20, 2010; accepted May 19, 2010

\begin{abstract}
The importance of epidemiology in our life has stimulated researchers to extend the classic Susceptibles-Infectives-Removed (SIR) model to sophisticated models by including more factors in order to give detailed transmission dynamics of epidemic diseases. However, the integration of the quarantine policy and age-structure is less addressed. In this work we propose an age-structured MSIQR (temporarily immune-susceptibles-infectives-quarantined-removed) model to study the $\mathfrak{R}_{0}>1$ impact of quarantine policies on the spread of epidemic diseases. Specifically, we investigate the existence of steady state solutions and stability property of the proposed model. The derived explicit expression of the basic reproductive number shows that the disease-free equilibrium is globally asymptotically stable if, and that the unique endemic equilibrium exists if. In addition, the stability conditions of the endemic equilibrium are derived.
\end{abstract}

Keywords: Epidemic Model, Quarantine, Age-Structure, Basic Reproductive Number, Endemic Equilibrium, Stability

\section{Introduction}

In recent years there has been increasing interest in the use of mathematical models for the analysis of real life epidemics. The need for accurate modelling of the epidemic process is vital, particularly because the financial consequences of infection disease outbreaks are growing. Two important recent examples are the 2001 foot and month disease outbreak in the UK [1] and the Severe Acute Respiratory Syndrome (SARS) epidemic in 2003 $[2,3]$. The fundamental model of the spread of epidemic desease was first derived by Kermack and MacKendrick [4] who studied the epidemic dynamics of an infectious disease in a population. In that model it is assumed that the population consists of three types of individuals: susceptibles (S), infectivies (I) and removed (R). The classic SIR model has influenced the study of epedimic diseases for many years. However, the simple assumption of the SIR model restricts its application to realistic problems. In recent years there have been extensive research interests to design more realistic models, including spatial models to address the spatial heterogenity on the the spatio-temporal patterns of disease dynamics [5,6]; stochastic models to study the influence of individuals with small population numbers and/or fluctuations of environment $[7,8]$; epidemic models with delay to describe the waiting-time between different compartments of the system $[9,10]$; and multi-scale models to investigate complex systems with multi-species [11].

Age is an important characteristic of population heterogeneity and age-structure plays a key role on the transmission dynamics of epidemic diseases. Individuals with different ages may have different contact rates, infection rates and survival capacities. Modern mathematical analysis of age-structured models started with Hoppensteadt [12,13] who developed epidemic models with both continuous chronological age and infection class age. Over the last three decades, the infection-agedependent epidemic models have been studied extensively and a number of mathematical models have been proposed to address different aspects related to age in epidemic transmission. Mathematical issues such as existence of steady state solutions as well as stability property have been analysis [14-20]. An excellent review 
regarding the epidemic modelling and age-structured epidemic modelling can be found in [21].

Quarantine is one of the age-old measures to control the spread of infectious diseases by isolating some infectives, in order to reduce transmissions of the infection to susceptibles. The word quarantine originally corresponded to a period of forty days, which is the length of time that arriving ships suspected of plague infection were constrained from intercourse with the shore in Mediterranean ports in the 15-19th centuries [22]. The word quarantine has evolved to mean forced isolation or stoppage of interactions with others. By separating exposed people and restricting their movements, quarantine can reduce the contact rate of possibly infected individuals before symptoms appear, and thus delay the spread of that disease [23]. Quarantine is an important topic of epidemic modelling and the prevention of the spread of SARS in 2003 raised great interests in mathematical modelling to investigate the effects of different quarantine policies [24,25]. A number of mathematical models have been developed to studying the quarantine intervention measures [26,27].

There has been accumulated evidence recently showing that there is considerable age-related variation in susceptibility and transmissibility with regrading to epidemic diseases for which different quarantine policies have been applied [28]. However, compared with the age-structured models or models for studying the effects of quarantine policies, the development of epidemic models for both age-structure and quarantine policies is still at the very early stage. In this work we will propose an age-structured MSIQR epidemic model, identify the basic reproduction number, find the disease-free endemic equilibria, and determine its stability. The remainder of this paper is organized as follows. In section 2 we will introduce the mathematical model. Section 3 discusses the reproduction number and stability of uninfected state. Then the existence of steady state solutions will be studied in Section 4. Finally,we will investigate the stability analysis for endemic equilibrium solutions in Section 5.

\section{Mathematical Model}

To study the effect of quarantine on the spread of endemic infectious diseases, we propose an age-structured MSIQR epidemic model by introducing a class of quarantined individuals into an age-structured MSEIS endemic model [18]. Here the quarantined people means those who have been removed and isolated either voluntarily or coercively from the infectious class. They could be people who choose to stay at home from school or work because they are sick for some milder diseases, or those who are forced into isolation for other more severe diseases. It is assumed that these quarantined individuals do not mix with others so that they do not infect susceptibles.

Before constituting an age-structured MSIQR epidemic model, we first introduce variables and notations. Here $a$ denotes the age of individuals, $t$ time, $a^{+}$ the highest age attained by the individuals in the population. We assume that the population is in a stationary demographic state, and divide a closed population into five compartments. Let $\mathrm{M}(a, t), \mathrm{S}(a, t), \mathrm{I}(a, t), \mathrm{Q}(a, t)$ and $\mathrm{R}(a, t)$ be the age-dependent densities of the temporarily immune, susceptible, infected, quarantine and immune (or removed) population, respectively, at time $t$. Then the total population density is

$$
\mathrm{N}(a, t)=\mathrm{M}(a, t)+\mathrm{S}(a, t)+\mathrm{I}(a, t)+\mathrm{Q}(a, t)+\mathrm{R}(a, t) .
$$

In this model it is assumed that the susceptible individuals, once being infected, will be moved into the infectious class; then the infected individuals may be selected for quarantine and thus transferred into the quarantine class; and finally people in the quarantine class will be transfered into the immune class after they have been recovered. Based on these assumptions, the spread of the disease can be described by a system of partial differential equations, given by

$$
\left\{\begin{array}{l}
\frac{\partial M(a, t)}{\partial t}+\frac{\partial M(a, t)}{\partial a} \\
=-(\mu(a)+d(a)) M(a, t) \\
\frac{\partial S(a, t)}{\partial t}+\frac{\partial S(a, t)}{\partial a} \\
=-(\mu(a)+\hat{\lambda}(a, t)) S(a, t)+d(a) M(a, t) \\
\frac{\partial I(a, t)}{\partial t}+\frac{\partial I(a, t)}{\partial a} \\
=-(\mu(a)+q(a)) I(a, t)+\hat{\lambda}(a, t) S(a, t) \\
\frac{\partial Q(a, t)}{\partial t}+\frac{\partial Q(a, t)}{\partial a} \\
=-(\mu(a)+\gamma(a)) Q(a, t)+q(a) I(a, t) \\
\frac{\partial R(a, t)}{\partial t}+\frac{\partial R(a, t)}{\partial a} \\
=-\mu(a) R(a, t)+\gamma(a)) Q(a, t)
\end{array}\right.
$$

where $\mu(a)$ denotes the average death rate of the population with age a and it is assumed that this rate is not affected by the presence of the disease; $d(a)$ is the rate for a renascent to become an susceptible individual. $\hat{\lambda}(a, t)$ is the age-dependent probability that a susceptible individual becomes infective individual at time t; $q(a)$ is the rate of an infective individual being quarantined, and $\gamma(a)$ is the recovery rate of the quarantined infective individuals. Here the age-dependent transmission 
coefficient $\hat{\lambda}(a, t)$ is defined by $\hat{\lambda}(a, t)=k(a) \lambda(t)$, where $k(a)$ denotes the age-dependent contact ratio and $\lambda(t)=\int_{0}^{a^{+}} \beta(a) \frac{I(a, t)}{N(a, t)} d a$, where $\beta(a)$ is the age dependent infection rate. Functions $k(a)$ and $\beta(a)$ satisfy the following properties

$$
\begin{aligned}
& k(a) \in L^{\infty}\left[0, a^{+}\right) ; k(a) \geq 0, \forall a \in\left[0, a^{+}\right), \\
& \beta(a) \in L^{\infty}\left[0, a^{+}\right) ; \beta(a) \geq 0, \forall a \in\left[0, a^{+}\right) .
\end{aligned}
$$

We use $b(a)$ to represent the age-dependent fertility rate and assume that this rate is not affected by the presence of the disease. Then the birth of individuals is defined by

$$
\begin{aligned}
& M(0, t)= \\
& \int_{0}^{a^{+}} b(a)[M(a, t)+S(a, t)+I(a, t)+Q(a, t)+R(a, t)] d a
\end{aligned} .
$$

It is assumed that any newly born individual is temporarily immune, namely

$$
S(0, t)=I(0, t)=Q(0, t)=R(0, t)=0 .
$$

In addition, the initial condition at $\mathrm{t}=0$ is denoted as

$$
\begin{aligned}
& M(a, 0)=M_{0}(a), S(a, 0)=S_{0}(a), I(a, 0)=I_{0}(a), \\
& Q(a, 0)=Q_{0}(a), R(a, 0)=R_{0}(a) .
\end{aligned}
$$

From system (1), it can be verified that the total population density satisfies the Mckendrick-von Forester population equation [19]

$$
\left\{\begin{array}{l}
\frac{\partial N(a, t)}{\partial t}+\frac{\partial N(a, t)}{\partial a}=-\mu(a) N(a, t), \\
N(0, t)=\int_{0}^{a^{+}} b(a) N(a, t) d a, \\
N(a, 0)=N_{0}(a)
\end{array}\right.
$$

where $\mathrm{N}_{0}(\mathrm{a})=\mathrm{M}_{0}(\mathrm{a})+\mathrm{S}_{0}(\mathrm{a})+\mathrm{I}_{0}(\mathrm{a})+\mathrm{Q}_{0}(\mathrm{a})+\mathrm{R}_{0}(\mathrm{a})$.

It is assumed that the birth rate and death rate satisfy

$$
\begin{gathered}
b(a) \in L^{\infty}\left[0, a^{+}\right) ; b(a) \geq 0, \forall a \in\left[0, a^{+}\right), \\
\mu(a) \in L_{L o c}^{1}\left[0, a^{+}\right) ; \mu(a) \geq 0, \forall a \in\left[0, a^{+}\right) . \\
\int_{0}^{a^{+}} \mu(\mathrm{a}) \mathrm{da}=+\infty . \text { In addition, b(a) }=0 \text { and } \mu(a)=0
\end{gathered}
$$

when the age a is above the maximal age $a^{+}$. Let $\Pi(a)$ is the survival function, defined by $\Pi(a)=e^{-\int_{0}^{a} \mu(\sigma) d \sigma}$, which is the proportion of individuals who survive to age a. Further, we assume that the net reproductive number is 1 , that is

$$
\int_{0}^{a^{+}} b(a) \prod(a) d a=1
$$

Then we have the relationship

$$
\mathrm{N}(\mathrm{a}, \mathrm{t})=\mathrm{N}_{\infty}(\mathrm{a})=\mathrm{b}(0) \prod(\mathrm{a}) .
$$

Furthermore, the initial conditions satisfy

$$
\left\{\begin{array}{l}
M_{0}(a) \geq 0, S_{0}(a) \geq 0, I_{0}(a) \geq 0, Q_{0}(a) \geq 0, R_{0}(a) \geq 0, \\
N_{\infty}(a)=M_{0}(a)+S_{0}(a)+I_{0}(a)+Q_{0}(a)+R_{0}(a) .
\end{array}\right.
$$

From (4) and (5), we have the following formula of the total renascent number

$$
b_{0}=\frac{\int_{0}^{a^{+}}\left[M_{0}(a)+S_{0}(a)+I_{0}(a)+Q_{0}(a)+R_{0}(a)\right] d a}{\int_{0}^{a^{+}} \prod(a) d a}
$$

Next we consider the fractions of the temporarily immune, susceptible, infected, quarantine and immune population at age a and time t, defined by

$$
\begin{aligned}
& U(a, t)=\frac{M(a, t)}{N_{\infty}(a)}, X(a, t)=\frac{S(a, t)}{N_{\infty}(a)}, Y(a, t)=\frac{I(a, t)}{N_{\infty}(a)}, \\
& Z(a, t)=\frac{Q(a, t)}{N_{\infty}(a)}, V(a, t)=\frac{R(a, t)}{N_{\infty}(a)} .
\end{aligned}
$$

Then system (1) can be written in a simpler form

$$
\begin{aligned}
& \frac{\partial U(a, t)}{\partial t}+\frac{\partial U(a, t)}{\partial a}=-d(a) U(a, t) \\
& \frac{\partial X(a, t)}{\partial t}+\frac{\partial X(a, t)}{\partial a}=-k(a) \lambda(t) X(a, t)+d(a) U(a, t) \\
& \left\{\frac{\partial Y(a, t)}{\partial t}+\frac{\partial Y(a, t)}{\partial a}=k(a) \lambda(t) X(a, t)-q(a) Y(a, t)\right. \\
& \frac{\partial Z(a, t)}{\partial t}+\frac{\partial Z(a, t)}{\partial a}=q(a) Y(a, t)-\gamma(a) Z(a, t) \\
& \frac{\partial V(a, t)}{\partial t}+\frac{\partial V(a, t)}{\partial a}=\gamma(a) Z(a, t) \\
& \lambda(t)=\int_{0}^{a^{+}} \beta(a) Y(a, t) d a, U(0, t)=1, X(0, t) \\
& =Y(0, t)=Z(0, t)=V(0, t)=0 \text {, } \\
& U(a, 0)=U_{0}(a), X(a, 0)=X_{0}(a), Y(a, 0) \\
& =Y_{0}(a), Z(a, 0)=Z_{0}(a) \text {, } \\
& V(a, 0)=V_{0}(a), 1 \\
& =U(a, t)+X(a, t)+Y(a, t)+Z(a, t)+V(a, t) .
\end{aligned}
$$

\section{Reproduction Number and Stability of Uninfected State}

In this work we are interested in the steady-state solution $(U(a), X(a), Y(a), Z(a), V(a))$ of system (7). In this case the steady state solution satisfies the following system of ordinary differential equations, given by 


$$
\begin{aligned}
& \left\{\begin{array}{l}
\frac{d U(a)}{d a}=-d(a) U(a) \\
\frac{d X(a)}{d a}=-k(a) \lambda X(a)+d(a) U(a) \\
\frac{d Y(a)}{d a}=k(a) \lambda X(a)-q(a) Y(a) \\
\frac{d Z(a)}{d a}=q(a) Y(a)-\gamma(a) Z(a) \\
\frac{d V(a)}{d a}=\gamma(a) Z(a)
\end{array}\right. \\
& \lambda=\int_{0}^{a^{+}} \beta(a) Y(a) d a, U(0)=1, X(0)=Y(0)=Z(0)
\end{aligned}
$$

It can be verified that system (8) has a non-illness solution $E_{0}:\left(U^{0}(a), X^{0}(a), Y^{0}(a), Z^{0}(a), V^{0}(a)\right)$ which is also a non-illness steady-state solution of system (7), satisfying

$$
\begin{aligned}
& U^{0}(a)=e^{-\int_{0}^{a} d(\sigma) d \sigma}, Y^{0}(a)=Z^{0}(a)=V^{0}(a)=0 . \\
& X^{0}(a)=\int_{0}^{a} d(\tau) e^{-\int_{0}^{\tau} d(\sigma) d \sigma} d \tau=1-U^{0}(a),
\end{aligned}
$$

In order to investigate the local stability of the nonillness steady-state solution, we linearize system (7) at $E_{0}$ by introducing the following variables

$$
\begin{aligned}
& U(a, t)=u(a, t)+U^{0}(a), X(a, t)=x(a, t)+X^{0}(a), \\
& Y(a, t)=y(a, t)+Y^{0}(a), Z(a, t) \\
& =z(a, t)+Z^{0}(a), V(a, t)=v(a, t)+V^{0}(a),
\end{aligned}
$$

Then the system (7) is transfered into a linearized system, given by

$$
\left\{\begin{array}{c}
\frac{\partial u(a, t)}{\partial t}+\frac{\partial u(a, t)}{\partial a}=-d(a) u(a, t) \\
\frac{\partial x(a, t)}{\partial t}+\frac{\partial x(a, t)}{\partial a}=-k(a) \tilde{\lambda}(t) X^{0}(a)+d(a) u(a, t) \\
\frac{\partial y(a, t)}{\partial t}+\frac{\partial y(a, t)}{\partial a}=k(a) \tilde{\lambda}(t) X^{0}(a)-q(a) y(a, t) \\
\frac{\partial z(a, t)}{\partial t}+\frac{\partial z(a, t)}{\partial a}=q(a) y(a, t)-\gamma(a) z(a, t) \\
\frac{\partial v(a, t)}{\partial t}+\frac{\partial v(a, t)}{\partial a}=\gamma(a) z(a, t) \\
\tilde{\lambda}(t)=\int_{0}^{a^{+}} \beta(a) y(a, t) d a, u(0, t)=1, \\
x(0, t)=y(0, t)=z(0, t)=v(0, t)=0 .
\end{array}\right.
$$

Now we are looking for exponential solutions of system (11), i.e., solutions of the form

$$
\begin{aligned}
& u(a, t)=\bar{u}(a) e^{\omega t}, x(a, t)=\bar{x}(a) e^{\omega t}, y(a, t)=\bar{y}(a) e^{\omega t}, \\
& z(a, t)=\bar{z}(a) e^{\omega t}, v(a, t)=\bar{v}(a) e^{\omega t},
\end{aligned}
$$

where age-dependent functions $\bar{u}(a), \bar{x}(a), \bar{y}(a), \bar{z}(a)$ and $\bar{v}(a)$ as well as parameter $\omega$ satisfy

$$
\begin{gathered}
\bar{\lambda}(t)=\int_{0}^{a^{+}} \beta(a) \bar{y}(a) d a, \bar{u}(0)=e^{-\omega t}, \\
\bar{x}(0)=\bar{y}(0)=\bar{z}(0)=\bar{v}(0)=0 . \\
\left\{\begin{array}{l}
\frac{d \bar{u}(a)}{d a}=-(d(a)+\omega) \bar{u}(a) \\
\frac{d \bar{x}(a)}{d a}=-\omega \bar{x}(a)+d(a) \bar{u}(a)-\bar{\lambda} k(a) X^{0}(a) \\
\frac{d \bar{y}(a)}{d a}=\bar{\lambda} k(a) X^{0}(a)-(q(a)+\omega) \bar{y}(a) \\
\frac{d \bar{z}(a)}{d a}=-(\gamma(a)+\omega) \bar{z}(a)+q(a) \bar{y}(a) \\
\frac{d \bar{v}(a)}{d a}=-\omega \bar{v}(a)+\gamma(a) \bar{z}(a)
\end{array}\right.
\end{gathered}
$$

From the third equation of (12), we have

$$
\bar{y}(a)=\bar{\lambda} \int_{0}^{a} k(\tau) e^{-\int_{\tau}^{a} q(\sigma) d \sigma} e^{-\omega(a-\tau)} X^{0}(\tau) d \tau .
$$

After substituting (13) into the expression of $\bar{\lambda}(t)$, we have

$$
\bar{\lambda}=\int_{0}^{a^{+}} \beta(a)\left[\bar{\lambda} \int_{0}^{a} k(\tau) e^{-\int_{\tau}^{a} q(\sigma) d \sigma} e^{-\omega(a-\tau)} X^{0}(\tau) d \tau\right] d a .
$$

After dividing both sides of Equation (14) by $\bar{\lambda}(\bar{\lambda} \neq 0)$, we obtain a characteristic equation about the eigenvalue $\omega$

$$
F(\omega)=\int_{0}^{a^{+}} \beta(a)\left[\int_{0}^{a} k(\tau) e^{-\int_{\tau}^{a} q(\sigma) d \sigma} e^{-\omega(a-\tau)} X^{0}(\tau) d \tau\right] d a=1 .
$$

Here we define the basic reproduction number as $\mathfrak{R}_{0}=F(0)$. After substituting the explicit expression of $X^{0}(\tau)$ in (9) into (15) and interchanging the order of integrals, we obtain

$\mathfrak{R}_{0}=\int_{0}^{a^{+}} \beta(a)\left\{\int_{0}^{a} d(\tau) e^{-\int_{0}^{\tau} d(\sigma) d \sigma}\left[\int_{\tau}^{a} k(s) e^{-\int_{s}^{a} q(\sigma) d \sigma} d s\right] d \tau\right\} d a$.

Theorem 1. The uninfected state $E_{0}$ is linearly stable if $\mathfrak{R}_{0}<1$ and it is linearly unstable if $\mathfrak{R}_{0}>1$. Proof. It is assumed that $\omega$ is a real number. From (15), we have $F^{\prime}(\omega)<0, \lim _{\omega \rightarrow+\infty} F(\omega)=0, \lim _{\omega \rightarrow-\infty} F(\omega)=+\infty$.

Then $F(\omega)$ is a strictly monotone decreasing function at $(-\infty,+\infty)$, iff there is an unique negative real number root $\omega^{*}$ of (15) when $\mathfrak{R}_{0}<1$ (namely $\mathrm{F}(0)<$ $1)$. In addition, there is a unique positive real root of (15) iff $\mathfrak{R}_{0}>1$.

In the following, we will prove that $\omega^{*}$ is a real 
component dominate solution of $F(\omega)=1$. Firstly, we suppose that $\omega=\xi+\eta i$ is an arbitrary plural root of (15) and note that $1=|F(\omega)|=|F(\xi+\eta i)| \leq F(\xi)$.

That is $F\left(\omega^{*}\right) \leq F(\xi)$.

Thus we have shown that $\xi<\omega^{*}$. Therefore we have $R \omega \leq \omega^{*}$. From above, we know that there is only one negative real component root of (15) when $\mathfrak{R}_{0}<1$. In addition, there is a positive real component root of (15) if $\mathfrak{R}_{0}>1$. That is, when $\mathfrak{R}_{0}<1$, uninfected state $E_{0}$ is locally asymptotically stable; when $\mathfrak{R}_{0}>1$, uninfected state $E_{0}$ is unstable.

Theorem 2. When $\mathfrak{R}_{0}<1$, the uninfected state $E_{0}\left(U^{0}(a), X^{0}(a), Y^{0}(a), Z^{0}(a), V^{0}(a)\right)$ is globally asymptotically stable.

Proof. In order to prove that $E_{0}$ is globally asymptotically stable, we need to confirm that, when $t \rightarrow \infty$, the following limits hold,

$$
\begin{aligned}
& U(a, t) \rightarrow U^{0}(a), U(a, t) \rightarrow U^{0}(a), X(a, t) \rightarrow \\
& X^{0}(a), Y(a, t) \rightarrow 0, Z(a, t) \rightarrow 0, V(a, t) \rightarrow 0 .
\end{aligned}
$$

By integrating the first equation of (7) along the characteristic curves, we have

$$
U(a, t)=\left\{\begin{array}{l}
e^{-\int_{0}^{a} d(\xi) d \xi}, a \leq t \\
U_{0}(a-t) e^{-\int_{0}^{t} d(a-\xi) d \xi}, a>t
\end{array}\right.
$$

Note that $U(a, t)=U^{0}(a)$ when $a \leq t$.

Similarly after integrating the second and third equations of (7) along the characteristic curves, we obtain

$$
X(a, t)=\left\{\begin{array}{l}
\int_{0}^{a} d(\tau) e^{-\int_{\tau}^{a} k(\sigma) \lambda(\sigma-a+t) d \sigma} U(\tau, \tau+t-a) d \tau, a \leq t \\
X_{0}(a-t) e^{-\int_{0}^{t} k(a-\tau) \lambda(t-\tau) d \tau}+\int_{0}^{t} d(a-\tau) . \\
U(a-\tau, t-\tau) e^{-\int_{0}^{\tau} k(a-\sigma) \lambda(t-\sigma) d \sigma} d \tau, a>t
\end{array}\right.
$$

$$
\begin{aligned}
& Y(a, t)= \\
& \left\{\begin{array}{l}
\int_{0}^{a} k(\tau) \lambda(t-a+\tau) e^{-\int_{\tau}^{a} q(\sigma) d \sigma} X(\tau, \tau+t-a) d \tau, a \leq t \\
Y_{0}(a-t) e^{-\int_{0}^{t} q(a-\tau) d \tau}+\int_{0}^{t} k(a-\tau) \lambda(t-\tau) \\
\cdot e^{-\int_{0}^{\tau} q(a-\sigma) d \sigma} X(a-\tau, t-\tau) d \tau, a>t
\end{array}\right.
\end{aligned}
$$

By substituting (18) into (19) and interchanging the integral order, we obtain, when $a \leq t$,

$$
Y(a, t)=\int_{0}^{a} d(\tau) U(\tau, \tau+t-a) G(a, \tau) d \tau
$$

where

$G(a, t)=\int_{\tau}^{a} k(s) \lambda(t-a+s) e^{-\int_{s}^{a} q(\sigma) d \sigma} e^{-\int_{\tau}^{s} k(\sigma) \lambda(\sigma+t-a) d \sigma} d s$.

By substituting (20) into the expression of $\tilde{\lambda}(t)$, we derive, when $a \leq t$,

$$
\begin{aligned}
& \tilde{\lambda}(t)=\int_{0}^{t} \beta(a)\left\{\int_{0}^{a} d(\tau) U(\tau, \tau+t-a) G(a, \tau) d \tau\right\} d a \\
& +\int_{t}^{a^{+}} \beta(a) Y(a, t) d a .
\end{aligned}
$$

Notice that $Y(a, t) \leq 1$. It is clear that the second integral in (21) is less than $\int_{t}^{a^{+}} \beta(a) d a$. When $t \rightarrow \infty$, we obtain $\int_{t}^{a^{+}} \beta(a) d a \rightarrow 0$.

Notice that

$$
\begin{aligned}
& U(a, t)=1-X(a, t)-Y(a, t)-Z(a, t)- \\
& V(a, t) \leq 1-X(a, t)=1-X^{0}(a)(a<t) .
\end{aligned}
$$

From the above statement, we have

$$
\begin{aligned}
& \tilde{\lambda}(t) \leq \int_{0}^{t} \beta(a)\left\{\int_{0}^{a} d(\tau)\left(1-X^{0}(\tau)\right) G(a, \tau) d \tau\right\} d a \\
& +\int_{t}^{a^{+}} \beta(a) d a .
\end{aligned}
$$

By making limitation on both sides of above equation, we obtain $\limsup \lambda(t) \leq \mathfrak{R}_{0} \limsup \lambda(t)$. When $\mathfrak{R}_{0}<1$, we have $\limsup \lambda(t)=0$.

From (20), we have $\limsup Y(a, t)=0$.

From the fourth equation of (7), we obtain, when $\mathrm{t}>\mathrm{a}$, $Z(a, t)=\int_{0}^{a} q(\tau) Y(\tau, \tau+t-a) e^{-\int_{\tau}^{a} \gamma(\sigma) d \sigma} d \tau$ and $\quad \limsup _{t \rightarrow \infty}$ $Z(a, t)=0$.

By integrating the fifth equation of (7), we obtain, when $\mathrm{t}>\mathrm{a}, \quad V(a, t)=\int_{0}^{a} \gamma(\tau) Z(\tau, \tau+t-a) d \tau$ and $\limsup V(a, t)=0$.

By making limitation on (17) and (18), we have

$$
\begin{aligned}
& \limsup _{t \rightarrow \infty} U(a, t)=e^{-\int_{0}^{a} d(\sigma) d \sigma}=U^{0}(a), \\
& \limsup X(a, t)=1-U^{0}(a) .
\end{aligned}
$$

Therefore, when $\mathfrak{R}_{0}<1$, the disease-free equilibrium is globally asymptotically stable.

\section{Existence of Steady State Solutions}

After showing that the uninfected equilibrium is unstable at $\mathfrak{R}_{0}>1$ in Theorem 2, we will prove that there is an endemic equilibrium of system (7) under the same conditions, that is, there is a time-independent solution 


$$
E^{*}:\left(U^{*}(a), X^{*}(a), Y^{*}(a), Z^{*}(a), V^{*}(a)\right)
$$

where $Y^{*}(a) \neq 0, Z^{*}(a) \neq 0, V^{*}(a) \neq 0$.

Theorem 3. When $\mathfrak{R}_{0}>1$, there is an unique endemic equilibrium solution of system (7).

Proof. Let $E^{*}$ be a steady-state solution for Equation (7). Then it can be verified that the solution satisfies

$$
\left\{\begin{array}{l}
\frac{d U^{*}(a)}{d a}=-d(a) U^{*}(a) \\
\frac{d X^{*}(a)}{d a}=d(a) U^{*}(a)-\lambda^{*} k(a) X^{*}(a) \\
\frac{d Y^{*}(a)}{d a}=\lambda^{*} k(a) X^{*}(a)-q(a) Y^{*}(a) \\
\frac{d Z^{*}(a)}{d a}=-\gamma(a) Z^{*}(a)+q(a) Y^{*}(a) \\
\frac{d V^{*}(a)}{d a}=\gamma(a) Z^{*}(a)
\end{array}\right.
$$

$\lambda^{*}=\int_{0}^{a^{+}} \beta(a) Y^{*}(a) d a, U^{*}(0)=1, X^{*}(0)=Y^{*}(0)=Z^{*}(0)$

$=V^{*}(0)=0,1=U^{*}(a)+X^{*}(a)+Y^{*}(a)+Z^{*}(a)+V^{*}(a)$.

Then the solution of the above system is

$$
\begin{aligned}
U^{*}(a) & =e^{-\int_{0}^{a} d(\sigma) d \sigma} \\
X^{*}(a) & =\int_{0}^{a} d(\tau) U^{*}(\tau) e^{-\int_{\tau}^{a} \lambda^{*} k(\sigma) d \sigma} d \tau \\
Y^{*}(a) & =\lambda^{*} \int_{0}^{a} k(\tau) X^{*}(\tau) e^{-\int_{\tau}^{a} q(\sigma) d \sigma} d \tau \\
Z^{*}(a) & =\int_{0}^{a} q(\tau) Y^{*}(\tau) e^{-\int_{\tau}^{a} \gamma(\sigma) d \sigma} d \tau \\
V^{*}(a) & =\int_{0}^{a} \gamma(\tau) Z^{*}(\tau) d \tau
\end{aligned}
$$

By substituting (24) into (25), we obtain

$$
X^{*}(a)=\int_{0}^{a} d(\tau) e^{-\int_{0}^{\tau} d(\sigma) d \sigma} e^{-\int_{\tau}^{a} \lambda^{*} k(\sigma) d \sigma} d \tau
$$

By substituting (29) into (26) and exchanging the order of integration, we obtain

$$
\begin{aligned}
& Y^{*}(a)=\lambda^{*} \int_{0}^{a} d(\tau) e^{-\int_{0}^{\tau} d(\sigma) d \sigma}\left\{\int_{\tau}^{a} k(\eta) e^{-\int_{\eta}^{a} q(\sigma) d \sigma} d \eta\right\} \\
& \cdot e^{-\int_{\tau}^{a} \lambda^{*} k(\sigma) d \sigma} d \tau
\end{aligned}
$$

By substituting (29) into the expression of $\lambda^{*}(t)$, we have

$$
\begin{aligned}
& \lambda^{*}=\int_{0}^{a^{+}} \beta(a)\left\{\lambda^{*} \int_{0}^{a} d(\tau) e^{-\int_{0}^{\tau} d(\sigma) d \sigma}\left[\int_{\tau}^{a} k(\eta) e^{-\int_{\eta}^{a} q(\sigma) d \sigma} d \eta\right]\right. \\
& \left.\cdot e^{-\int_{\tau}^{a} \lambda^{*} k(\sigma) d \sigma} d \tau\right\} d a .
\end{aligned}
$$

By dividing both sides of the above equation by $\lambda^{*}\left(\lambda^{*} \neq 0\right)$, we obtain the characteristic equation for the eigenvalue $\lambda^{*}$, given by

$$
\begin{aligned}
& H\left(\lambda^{*}\right)=\int_{0}^{a^{+}} \beta(a)\left\{\int_{0}^{a} d(\tau) e^{-\int_{0}^{\tau} d(\sigma) d \sigma}\right. \\
& \left.\left[\int_{\tau}^{a} k(\eta) e^{-\int_{\eta}^{a} q(\sigma) d \sigma} d \eta\right] e^{-\int_{\tau}^{a} \lambda^{*} k(\sigma) d \sigma} d \tau\right\} d a=1 .
\end{aligned}
$$

It is clear that $H\left(\lambda^{*}\right)$ is continuous function of $\lambda^{*}$. If $\lambda^{*}=0$, we have $Y^{*}(a)=Z^{*}(a)=V^{*}(a)=0$. Thus $E^{*}$ is an uninfected equilibrium solution.

From (30) and (31), we have $H(0)=\mathfrak{R}_{0}$. If $\mathfrak{R}_{0}>1$, then we have $H(0)>1$.

From (30) and $Y^{*}(a)<1$, we obtain

$\lambda^{*} \int_{0}^{a} d(\tau) e^{-\int_{0}^{\tau} d(\sigma) d \sigma}\left[\int_{\tau}^{a} k(\eta) e^{-\int_{\eta}^{a} \eta(\sigma) d \sigma} d \eta\right] e^{-\int_{\tau}^{a} \lambda^{*} k(\sigma) d \sigma} d \tau<1$.

From (31) and (32), we obtain, when $\lambda^{*}>0$,

$$
\lambda^{*} H\left(\lambda^{*}\right)=\int_{0}^{a^{+}} \beta(a) Y^{*}(a) d a<\int_{0}^{a^{+}} \beta(a) d a .
$$

Let $\beta^{+}=\int_{0}^{a^{+}} \beta(a) d a$, thus $\lambda^{*} H\left(\lambda^{*}\right)<\beta^{+}$. If $\lambda^{*}=\beta^{+}$, we have $H\left(\beta^{+}\right)<1$. By using $\mathrm{H}(0)>1$ and the expression of $H\left(\lambda^{*}\right)$, we conclude that $H\left(\lambda^{*}\right)$ is monotone decreasing continuous function for $\lambda^{*}$. Thus, there is an unique solution $\hat{\lambda}^{*}$ of $H\left(\lambda^{*}\right)=1$ at $\left(0, \beta^{+}\right)$. Therefore, if $\mathfrak{R}_{0}>1$, there is an unique endemic equilibrium solution of system (7) which is defined by $\hat{\lambda}^{*}$ in (23).

\section{Stability of Endemic Equilibrium Solutions}

To investigate the local stability of the endemic equilibrium solutions $E^{*}$, we linearize system (7) at $E^{*}$ by introducing the following variables

$$
\begin{aligned}
U(a, t) & =u(a, t)+U^{*}(a), X(a, t) \\
& =x(a, t)+X^{*}(a), Y(a, t)=y(a, t)+Y^{*}(a), \\
Z(a, t) & =z(a, t)+Z^{*}(a), V(a, t) \\
& =v(a, t)+V^{*}(a), \lambda(t)=\hat{\lambda}(t)+\lambda^{*}
\end{aligned}
$$

By substituting these variables into system (7), we obtain its linearized equation at $E^{*}$, that is 


$$
\left\{\begin{array}{l}
\frac{\partial u(a, t)}{\partial t}+\frac{\partial u(a, t)}{\partial a} \\
=-d(a) u(a, t) \\
\frac{\partial x(a, t)}{\partial t}+\frac{\partial x(a, t)}{\partial a} \\
\quad=-k(a) \lambda^{*}(t) x(a, t)-k(a) \hat{\lambda}(t) X^{*}(a)+d(a) u(a, t) \\
\frac{\partial y(a, t)}{\partial t}+\frac{\partial y(a, t)}{\partial a} \\
=k(a) \lambda^{*}(t) x(a, t)+k(a) \tilde{\lambda}(t) X^{*}(a)-q(a) y(a, t) \\
\frac{\partial z(a, t)}{\partial t}+\frac{\partial z(a, t)}{\partial a} \\
=q(a) y(a, t)-\gamma(a) z(a, t) \\
\frac{\partial v(a, t)}{\partial t}+\frac{\partial v(a, t)}{\partial a} \\
=\gamma(a) z(a, t)
\end{array}\right.
$$$$
\hat{\lambda}(t)=\int_{0}^{a^{+}} \beta(a) y(a, t) d a, \lambda^{*}=\int_{0}^{a^{+}} \beta(a) Y^{*}(a) d a .
$$

Now we look for exponential solutions of (33), i.e., solutions of the form

$$
\begin{aligned}
& u(a, t)=\bar{u}(a) e^{\lambda t}, x(a, t)=\bar{x}(a) e^{\lambda t}, y(a, t)=\bar{y}(a) e^{\lambda t}, \\
& z(a, t)=\bar{z}(a) e^{\lambda t}, v(a, t)=\bar{v}(a) e^{\lambda t} \\
& \left\{\begin{array}{l}
\frac{d \bar{u}(a)}{d a}=-(d(a)+\lambda) \bar{u}(a) \\
\frac{d \bar{x}(a)}{d a}=d(a) \bar{u}(a)-\left(\lambda+\lambda^{*} k(a)\right) \bar{x}(a)-\vec{\lambda} k(a) X^{*}(a) \\
\frac{d \bar{y}(a)}{d a}=-(\lambda+q(a)) \bar{y}(a)+\lambda^{*} k(a) \bar{x}(a)+\vec{\lambda} k(a) X^{*}(a) \\
\frac{d \bar{z}(a)}{d a}=-(\lambda+\gamma(a)) \bar{z}(a)+q(a) \bar{y}(a) \\
\frac{d \bar{v}(a)}{d a}=-\lambda \bar{v}(a)+\gamma(a) \bar{z}(a)
\end{array}\right.
\end{aligned}
$$

$$
\begin{aligned}
& \vec{\lambda}(t)=\int_{0}^{a^{+}} \beta(a) \bar{y}(a) d a, \lambda^{*}=\int_{0}^{a^{+}} \beta(a) Y^{*}(a) d a, \\
& \bar{u}(0)=0, \bar{x}(0)=\bar{y}(0)=\bar{z}(0)=\bar{v}(0)=0 .
\end{aligned}
$$

By using the assumption that $U^{*}(0)=1$, we have $\bar{u}(0)=0$ from $u(0, t)=\bar{u}(0) e^{\lambda t}=0$. Then from the first equation of (35), we have $\bar{u}(a)=0$.

Suppose $\vec{\lambda} \neq 0$ and let

$$
x(a)=\frac{\bar{x}(a)}{\vec{\lambda}}, y(a)=\frac{\bar{y}(a)}{\vec{\lambda}}, z(a)=\frac{\bar{z}(a)}{\vec{\lambda}}, v(a)=\frac{\bar{v}(a)}{\vec{\lambda}}
$$

at $a \in\left(0, a^{+}\right)$, then (35) can be written as

$$
\left\{\begin{aligned}
& \frac{d x(a)}{d a}=-\left(\lambda^{*} k(a)+\lambda\right) x(a)-k(a) X^{*}(a) \\
& \frac{d y(a)}{d a}=-(q(a)+\lambda) y(a)+\lambda^{*} k(a) x(a)+k(a) X^{*}(a) \\
& \frac{d z(a)}{d a}=-(\lambda+\gamma(a)) z(a)+q(a) y(a) \\
& \frac{d v(a)}{d a}=-\lambda v(a)+\gamma(a) z(a) \\
& 1=\int_{0}^{a^{+}} \beta(a) y(a) d a, \lambda^{*}=\int_{0}^{a^{+}} \beta(a) Y^{*}(a) d a, \\
& x(0)=y(0)=z(0)=v(0)=0 .
\end{aligned}\right.
$$

From (36) and specifically

$$
x(0)=y(0)=z(0)=v(0)=0,
$$

we obtain

$$
x(a)+y(a)+z(a)+v(a)=0 .
$$

and the solution of (36) is represented by

$$
\begin{aligned}
& x(a)=-\int_{0}^{a} k(\tau) X^{*}(\tau) e^{-\int_{\tau}^{a}\left(\lambda+\lambda^{*} k(\sigma)\right) d \sigma} d \tau \\
& y(a)=\int_{0}^{a}\left[k(\tau) X^{*}(\tau)+\lambda^{*} k(\tau)\right. \\
&\cdot x(\tau)] e^{-\lambda(a-\tau)} e^{-\int_{\tau}^{a} q(\sigma) d \sigma} d \tau \\
& z(a)=\int_{0}^{a} q(\tau) y(\tau) e^{-\lambda(a-\tau)} e^{-\int_{\tau}^{a} \gamma(\sigma) d \sigma} d \tau \\
& v(a)=\int_{0}^{a} \gamma(\tau) z(\tau) e^{-\lambda(a-\tau)} d \tau
\end{aligned}
$$

where $X^{*}(\tau)=\int_{0}^{\tau} d(\eta) U^{*}(\eta) e^{-\int_{\tau}^{\eta} \lambda^{*} k(\sigma) d \sigma} d \eta$

By substituting (38) into (39), we have

$$
\begin{aligned}
y(a) & =\int_{0}^{a} k(\tau)\left[X^{*}(\tau) e^{-\lambda(a-\tau)}-\lambda^{*} \int_{0}^{\tau} k(\eta)\right. \\
& \left.\cdot X^{*}(\eta) e^{-\lambda(a-\eta)} e^{-\int_{\eta}^{\tau} \lambda^{*} k(\sigma) d \sigma} d \eta\right] e^{-\int_{\tau}^{a} q(\sigma) d \sigma} d \tau
\end{aligned}
$$

By substituting (43) into the next equation of (36), we have

$$
\begin{aligned}
& 1=\int_{0}^{a^{+}} \beta(a) y(a) d a=\int_{0}^{a^{+}} \beta(a)\left\{e ^ { - \int _ { \tau } ^ { a } q ( \sigma ) d \sigma } d \tau \int _ { 0 } ^ { a } k ( \tau ) \left[X^{*}(\tau)\right.\right. \\
& \left.\left.\cdot e^{-\lambda(a-\tau)}-\lambda^{*} \int_{0}^{\tau} k(\eta) X^{*}(\eta) e^{-\lambda(a-\eta)} e^{-\int_{\eta}^{\tau} \lambda^{*} k(\sigma) d \sigma} d \eta\right]\right\} d a
\end{aligned}
$$

Supposing that the right side of (44) is $Q(\lambda)$, that is

$$
Q(\lambda)=\int_{0}^{a^{+}} \beta(a) y(a) d a=1
$$

1) Firstly we will prove that $Q(\lambda) \geq 0$ and $Q(\lambda)$ is monotone decreasing function for $\lambda$ as well as $Q(\lambda) \rightarrow 0$ at $\lambda \rightarrow+\infty$.

By exchanging the integral order in $Q(\lambda)$ we obtain 


$$
Q(\lambda)=\int_{0}^{a^{+}} \beta(a)\left[\int_{0}^{a} k(\tau) X^{*}(\tau) e^{-\lambda(a-\tau)} f(a, \tau) d \tau\right] d a .
$$

where

$$
f(a, \tau)=e^{-\int_{\tau}^{a} q(\sigma) d \sigma}-\lambda^{*} \int_{\tau}^{a} k(\eta) e^{-\int_{\eta}^{a} q(\sigma) d \sigma} e^{-\int_{\tau}^{\eta} \lambda^{*} k(\sigma) d \sigma} d \eta
$$

and $f(a, \tau) \geq 0$ when $0 \leq \tau \leq a \leq a^{+}$. Now we have proved that $Q(\lambda) \geq 0$. Obviously, $Q(\lambda)$ is monotone decreasing function for $\lambda$ and $Q(\lambda) \rightarrow 0$ at $\lambda \rightarrow+\infty$.

2) From Equation (31), we have

$$
\int_{0}^{a^{+}} \beta(a)\left[\int_{0}^{a} k(\tau) X^{*}(\tau) e^{-\int_{\tau}^{a} q(\sigma) d \sigma} d \tau\right] d a=1 .
$$

Thus

$$
Q(0)=\int_{0}^{a^{+}} \beta(a)\left[\int_{0}^{a} k(\tau) X^{*}(\tau) e^{-\int_{\tau}^{a} q(\sigma) d \sigma} d \tau\right] d a-\Gamma
$$

where

$$
\begin{aligned}
\Gamma & =\lambda^{*} \int_{0}^{a^{+}} \beta(a)\left\{\int _ { 0 } ^ { a } k ( \tau ) \left[\int_{0}^{\tau} k(\eta) X^{*}(\eta)\right.\right. \\
& \left.\left.\cdot e^{-\int_{\eta}^{\tau} \lambda^{*} k(\sigma) d \sigma} d \eta\right] e^{-\int_{\tau}^{a} q(\sigma) d \sigma} d \tau\right\} d a>0 .
\end{aligned}
$$

From Equation (46), we derive that $Q(0)<1$. Therefore from 1) and 2), we conclude that the solution $\lambda$ of $Q(\lambda)=1$ is negative.

If $\mathfrak{R}_{0}>1$, there is only one negative real component root of the characteristic Equation (45), that is, the endemic equilibrium solution is locally asymptotically stable. Finally, we have the following theorem.

Theorem 4. If $\mathfrak{R}_{0}>1$ and

$$
\begin{aligned}
& f(a, \tau) \\
& =e^{-\int_{\tau}^{a} q(\sigma) d \sigma}-\lambda^{*} \int_{\tau}^{a} k(\eta) e^{-\int_{\eta}^{a} q(\sigma) d \sigma} e^{-\int_{\tau}^{\eta} \lambda^{*} k(\sigma) d \sigma} d \eta \geq 0,
\end{aligned}
$$

the endemic equilibrium $E^{*}$ is locally asymptotically stable.

\section{Conclusions}

In this paper, we have proposed an age-structured MSIQR epidemic model. This is the first approach to investigate the combined effect of quarantine policies and age-structure on the transmission dynamics of epidemic diseases. We have derived the sufficient condition under which the disease-free equilibrium is globally asymptotically stable or the unique endemic equilibrium exists. Furthermore, we have provided the stability conditions of the endemic equilibrium.

\section{References}

[1] N. M. Ferguson, C. A. Donnelly and R. M. Anderson, "Transmission Intensity and Impact of Control Policies on the Foot and Mouth Epidemic in Great Britain,” Nature, Vol. 413, No. 6855, 2001, pp. 542-548.

[2] M. Lipsitch, T. Cohen, B. Cooper, J. M. Robins, S. Ma, L. James, G. Gopalakrishna, S. K. Chew, C. C. Tan, M. H. Samore, D. Fisman and M. Murray, "Transmission Dynamics and Control of Severe Acute Respiratory Syndrome,” Science, Vol. 300, No. 5627, 2003, pp. 19661970.

[3] S. Hsu and L. W. Roeger, "The Final Size of a SARS epidemic Model without Quarantine,” Journal of Mathematical Analysis and Applications, Vol. 333, No. 2, 2007, pp. 557-566.

[4] W. O. Kermack and A. G. Mckendrick, "A Contribution to the Mathematical Theory of Epidemics," Proceedings of the Royal Society of London - Series A, Vol. 115, No. 772, 1927, pp. 700-721.

[5] S. Riley, "Large-Scale Spatial-Transmission Models of Infectious Disease,” Science, Vol. 316, No. 5829, 2007, pp. 1298-1301.

[6] P. E. Parham, B. K. Singh and N. M. Ferguson, “Analytic Approximation of Spatial Epidemic Models of Foot and Mouth Disease,” Theoretical Population Biology, Vol. 73, No. 3, 2008, pp. 349-368.

[7] M. J. Keeling, M. E. J. Woolhouse, D. J. Shaw, L. Matthews, M. Chase-Topping, D. T. Haydon, S. J. Cornell, J. Kappey, J. Wilesmith and B. T. Grenfell, "Dynamics of the 2001 UK Foot and Mouth Epidemic: Stochastic Dispersal in a Heterogeneous Landscape,” Science, Vol. 294, No. 5543, 2001, pp. 813-817.

[8] H. C. Tuckwell and R. J. Williams, "Some Properties of a Simple Stochastic Epidemic Model of SIR Type,” Mathematical Biosciences, Vol. 208, No. 1, 2007, pp. 76-97.

[9] S. Gao, L. Chen and Z. Teng, "Impulsive Vaccination of an SEIRS Model with Time Delay and Varying Total Population Size,” Bulletin of Mathematical Biology, Vol. 69, No. 2, 2007, pp. 731-745.

[10] H. W. Hethcode, "The Mathematics of Infectious Diseases,” SIAM Review, Vol. 42, No. 4, 2000, pp. 599-653.

[11] J. Arino, J. R. Davis, D. Hartley, R. Jordan, J. M. Miller and P. van den Driessche, "A Multi-Species Epidemic Model with Spatial Dynamics," Mathematical Medicine and Biology, Vol. 22, No. 2, 2005, pp. 129-142.

[12] F. Hoppensteadt, “An Age Dependent Epidemic Model,” Journal of the Franklin Institute, Vol. 297, No. 5, 1974, pp. 325-333.

[13] F. Hoppensteadt, "Mathematical Theories of Populations: Demographics," Genetics and Epidemics, SIAM, Philadelphia, 1975.

[14] Z. Zhang and J. G. Peng, “A SIRS Epidemic Model with Infection-Age Dependence,” Journal of Mathematical Analysis and Applications, Vol. 331, No. 2, 2007, pp. 1396-1414.

[15] H. R. Thieme and C. Castillo-Chavez, "How may Infection Age-Dependent Infectivity Affect the Dynamics of HIV/AIDS?” SIAM Journal on Applied Mathematics, Vol. 53, No. 5, 1993, pp. 1447-1479.

[16] C. M. Kribs-Zaleta and M. Martcheva, "Vaccination Stra- 
tegies and backward Bifurcation in an Age-since-Infection Structured Model,” Mathematical Biosciences, Vol. 177178, 2002, pp. 317-332.

[17] H. Inaba and H. Sekine, "A Mathematical Model for Chagas Disease with Infection-Age-Dependent Infectivity,” Mathematical Biosciences, Vol. 190, No. 1, 2004, pp. 3969.

[18] B. Fang and X. Li, "Stability of an Age-Structured MSEIS Epidemic Model with Infectivity in Latent Period,” Acta Mathematicae Applicatae Sinica, Vol. 31, No. 1, 2008, pp. 110-125.

[19] G. B. Webb, “Theory of Nonlinear Age-Dependent Population Dynamics,” CRC Press, Boca Raton, 1985.

[20] J. Li, Y. C. Zhou, Z. Z. Ma and M. Hyman, "Epidemiological Models for Mutating Pathogens," SIAM Journal on Applied Mathematics, Vol. 65, No. 1, 2004, pp. 1-23.

[21] H. W. Hethcotevan and P. den Driessche, "Two SIS Epidemiologic Models with Delays,” Journal of Mathematical Biology, Vol. 40, No. 1, 2000, pp. 3-26.

[22] W. H. McNeill, "Plagues and Peoples," Updated Edition, Anchor, Garden City, 1976.

[23] A. Mandavilli, "SARS Epidemic Unmasks Age-Old
Quarantine Conundrum,” Nature Medicine, Vol. 9, No. 5, 2003, p. 487.

[24] B. Diamond, "SARS Spreads New Outlook on Quarantine Models," Nature Medicine, Vol. 9, No. 12, 2003, p. 1441.

[25] Y. H. Hsieh, C. C. King, C. W. Chen, M. S. Ho, S. B. Hsu and Y. C. Wu, "Impact of Quarantine on the 2003 SARS Outbreak: A Retrospective Modeling Study,” Journal of Theoretical Biology, Vol. 244, No. 4, 2007, pp. 729-736.

[26] L. Sattenspiel and D. A. Herring, "Simulating the Effect of Quarantine on the Spread of the 1918-19 Flu in Central Canada,” Bulletin of Mathematical Biology, Vol. 65, No. 1, 2003, pp. 1-26.

[27] H. W. Hethcote, Z. E. Ma and S. B. Liao, "Effects of Quarantine in Six Endemic Models for Infectious Diseases,” Mathematical Biosciences, Vol. 180, No. 1, 2002, pp. 141-160.

[28] C. T. Bauch, J. O. Lloyd-Smith, M. P. Coffee and A. P. Galvani, "Dynamically Modelling SARS and Other Newly Emerging Respiratory Illnesses: Past, Present and Future,” Epidemiology, Vol. 16, No. 6, 2005, pp. 791-801. 